\title{
Airway drug pharmacokinetics via analysis of exhaled breath condensate
}

\author{
Charles R. Esther Jr. ${ }^{a}$, Richard C. Boucher ${ }^{b}$, M. Ross Johnson ${ }^{c}$, John H. Ansede ${ }^{c}$, Karl H. $^{\circ}$ \\ Donn ${ }^{\mathrm{c}}$, Thomas G. O'Riordan ${ }^{\mathrm{d}}$, Andrew J. Ghio ${ }^{\mathrm{e}}$, and Andrew J. Hirsh ${ }^{\mathrm{c}}$ \\ aPediatric Pulmonology, University of North Carolina at Chapel Hill, Chapel Hill, NC 27599 \\ ${ }^{b}$ Cystic Fibrosis/Pulmonary Research Center, University of North Carolina at Chapel Hill, Chapel \\ Hill, NC 27599, USA \\ 'Parion Sciences Inc, Durham NC, 27713, USA \\ 'Gilead Sciences Inc., Seattle, WA, 98102 USA \\ eEnvironmental Protection Agency, Chapel Hill, NC 27599, USA
}

\begin{abstract}
Although the airway surface is the anatomic target for many lung disease therapies, measuring drug concentrations and activities on these surfaces poses considerable challenges. We tested whether mass spectrometric analysis of exhaled breath condensate (EBC) could be utilized to noninvasively measure airway drug pharmacokinetics and predicted pharmacological activities.

Mass spectrometric methods were developed to detect a novel epithelial sodium channel blocker (GS-9411/P-680), two metabolites, a chemically related internal standard, plus naturally occurring solutes including urea as a dilution marker. These methods were then applied to EBC and serum collected from four (Floridian) sheep before, during and after inhalation of nebulized GS-9411/ P-680. Electrolyte content of EBC and serum was also assessed as a potential pharmacodynamic marker of drug activity. Airway surface concentrations of drug, metabolites, and electrolytes were calculated from EBC measures using EBC:serum urea based dilution factors.
\end{abstract}

GS-9411/P-680 and its metabolites were quantifiable in the sheep EBC, with peak airway concentrations between 1.9-3.4 $\mu \mathrm{M}$ measured 1 hour after inhalation. In serum, only Metabolite \#1 was quantifiable, with peak concentrations $~ 60$-fold lower than those in the airway ( $45 \mathrm{nM}$ at 1 hour). EBC electrolyte concentrations suggested a pharmacological effect; but this effect was not statistical significant.

Analysis of EBC collected during an inhalation drug study provided a method for quantification of airway drug and metabolites via mass spectrometry. Application of this methodology could provide an important tool in development and testing of drugs for airways diseases.

(c) 2013 Elsevier Ltd. All rights reserved.

Corresponding Author: Charles R. Esther Jr., MD, PhD, Pediatric Pulmonology, 450-D MacNider, CB\#7217, University of North Carolina at Chapel Hill, Chapel Hill, NC 27599, Tel: 919 966-1055, Fax: 919 966-6179, Charles_Esther@med.unc.edu.

Conflicts of Interest

This study was funded by Gilead Sciences, Inc.

Publisher's Disclaimer: This is a PDF file of an unedited manuscript that has been accepted for publication. As a service to our customers we are providing this early version of the manuscript. The manuscript will undergo copyediting, typesetting, and review of the resulting proof before it is published in its final citable form. Please note that during the production process errors may be discovered which could affect the content, and all legal disclaimers that apply to the journal pertain. 


\section{Keywords}

exhaled breath condensate; pharmacokinetics; sodium channel; urea; sheep

\section{Introduction}

Chronic airway diseases such as cystic fibrosis and chronic obstructive pulmonary disease are important causes of morbidity and mortality in the developed world [1, 2]. Because much of the pathophysiology of these diseases is localized to the airways, the surface of the airway epithelium represents the anatomic target for many pharmacological therapies. While the therapeutic benefit of these pharmaceutical agents is highly dependent on their concentrations in the airway, accurately measuring airway drug concentrations poses significant challenges to drug development. Healthy subjects and those with mild disease typically cannot spontaneously expectorate sputum for airway studies. Bronchoalveolar lavage and sputum induction have been utilized to obtain airway samples for drug concentrations [3,4], but both methods involve technical and interpretation challenges that limit use. Furthermore, both lavage and sputum induction require installation of salt solutions to the airway that induce changes to the airway milieu that may alter concentrations of drugs and/or biomarkers of efficacy.

Exhaled breath condensate $(\mathrm{EBC})^{1}$ has emerged as a respiratory sample with advantages over sputum or lavage fluid for assessing airway drug pharmacokinetics. EBC collection is simple and non-invasive, making longitudinal assessments feasible without altering airway constituents. Furthermore, recent studies suggest that the aerosols incorporated within EBC arise from the small airways [5, 6], which is also the site of early airways disease pathophysiology and the anatomic target for many therapies [7, 8]. However, analysis of EBC does pose considerable challenges. Airway secretions are highly dilute in EBC (dilution factors often $>1: 10,000)[9,10]$, and highly sensitive methods are required to measure drugs and biomarkers at the low concentrations found in $\operatorname{EBC}[9,11,12]$.

Furthermore, the extent of dilution can vary considerably both between subjects and within a subject over time $[9,10,13]$, complicating analysis of longitudinal changes in airway concentrations from measured $\mathrm{EBC}$ values.

We have developed methods to overcome these limitations by using mass spectrometry (MS) to measure multiple small molecules and metabolites at the low concentrations found in EBC. Because mass spectrometry is highly versatile, simultaneous measurements of concentrations of drugs, naturally occurring biomarkers, and dilution markers such as urea can be made to measure the pharmacokinetics of drugs delivered to airway surfaces. Dilution factors calculated from EBC:serum urea ratios can be used to control for dilutional variability and determine the actual concentrations of drugs and other metabolites on airway surfaces [13].

To assess the feasibility of EBC based measurement of airway drug pharmacokinetics, we developed MS methods to measure the concentrations of an investigational sodium channel antagonist GS-9411/P-680 and its metabolites at the low concentrations predicted in EBC. We then applied these methods to measure the drug, its metabolites, urea, and other constituents of airway secretions within EBC and serum collected during an animal study of inhalation of GS-9411/P-680. A sheep model was chosen for this study because this large

\footnotetext{
${ }^{1}$ Abbreviations: EBC, exhaled breath condensate; MS, mass spectrometry; LC-MS/MS, liquid chromatography-tandem mass spectrometry; SRM, selected reaction monitoring; ICP-OES, inductively coupled plasma-optical emission spectrography; ICP-MS, inductively coupled plasma-mass spectrography; $\mathrm{Na}$, sodium; K, potassium.
} 
animal model has been used successfully in monitoring respiratory effects post aerosol dosing with pharmacologic agents $[14,15]$.

\section{Methods}

\subsection{EBC and serum collection}

The animal study was conducted at Mount Sinai Medical Center, in Miami, Florida and approved by an Institutional Animal Care and Use Committee (Protocol \# 11-08-A-04). Four adult female ewes (Florida Native breed) ranging from 2 to 6 years in age were attained from Fairmeadow Farms (Ocala, Florida). The procedures utilized for animal restraint and dose administration via naso-tracheal intubation were similar that described previously [16]. A total of $4 \mathrm{~mL}$ of $3 \mathrm{mM} \mathrm{GS}-9411 / \mathrm{P}-680$ in water was aerosolized using a raindrop nebulizer over 10-15 minutes and delivered to the sheep lungs through the endotracheal tube. EBC was collected via an RTube (Respiratory Research, Inc, Charlottesville, VA) connected to the exhalation loop of the endotracheal tube, with chiller tubes held at $-80^{\circ} \mathrm{C}$ until immediately prior to collection and maintained over dry ice for the 30 min collection. Each collection was captured from tidal breathing at predose, immediately after dose, and at 1, 2, 4, and 6 hour time points. EBC was extracted from the RTube via the manufacturer's instructions at stored at $-80^{\circ} \mathrm{C}$ until analysis. Serum was obtained through blood draws at predose and at 1 and 6 hours post dose time points.

\subsection{Liquid chromatography-tandem mass spectrometry (LC-MS/MS)}

All samples were analyzed on an LC-MS/MS system consisting of an Acquity sample manager with a Thermo-Finnigan triple quadrupole mass spectrometer. Chromatographic separation was achieved on a C18 UPLC column (Waters HSS T3 $2.1 \times 150 \mathrm{~mm}$ ) using gradients of 5-95\% methanol in $0.1 \%$ formic acid, with a run time of 10 minutes. The method included selected reaction monitoring transitions from parent to product ion for drug and its metabolites as described in the text, plus urea, adenosine, tyrosine, and phenylalanine as previously described $[10,11]$. Peak areas were determined using the Xcalibur software program, and signal for each compound was defined as the peak area ratio of an authentic compound to an internal standard. Concentrations were determined via standard curves run in parallel.

For EBC, samples were thawed and divided into two aliquots. $400 \mu \mathrm{l}$ was utilized for LCMS/MS and the remainder for electrolyte determination (described below). For each $400 \mu \mathrm{l}$ EBC sample, $20 \mu \mathrm{l}$ of an internal standard solution was added that contained stable isotopically labeled urea, purines, and amino acids as previously described $[10,11]$ plus 50 $\mathrm{nM}$ of drug internal standard. EBC samples plus internal standard solution were lyophilized to dryness overnight then resuspended in $20 \mu \mathrm{l}$ of 50:50 water:methanol solution prior to LC-MS/MS. $10 \mu \mathrm{l}$ was then injected onto the LC-MS/MS system.

For serum, samples were thawed and $5 \mu 1$ of a $20 x$ concentration internal standard solution was added to $95 \mu \mathrm{l}$ serum. $5 \mu \mathrm{l}$ was injected onto the LC-MS/MS system.

\subsection{Electrolyte analysis}

Sodium and potassium concentrations in serum and EBC were initially measured using inductively coupled plasma-optical emission spectrography (ICP-OES as previously described [10]). However, because EBC electrolyte concentrations were often below reliable quantification limits by ICP-OES, EBC sodium and potassium concentrations were quantified using a more sensitive Perkin Elmer Elan 6000 inductively coupled plasma mass spectrometer (ICP-MS). 


\subsection{Statistical analysis}

Changes in airway or serum electrolyte concentrations over time or airway and serum drug concentrations were assessed using linear regression, and Student's T-tests was used to assess differences between pre-dose and immediately post-dose time points. Statistical tests were performed using GraphPad Prism v5.0 (GraphPad Software, San Diego California, USA). Data are presented as mean \pm SEM.

\section{Results}

\subsection{Mass spectrometric detection of drug and metabolites}

Mass spectrometric methods were developed for the experimental epithelial sodium channel blocker GS-9411/P-680 as well as its primary metabolites (Metabolite \#1 and Metabolite \#2). An inactive analogue similar to GS-9411/P-680 was used as an internal standard. The primary molecular ion, primary breakdown product ions, and optimal collision energy to create product ions in tandem mass spectrometry were determined empirically for each compound via loop injection of $10 \mu \mathrm{M}$ concentrations in a triple-quadrupole mass spectrometer (Table 1). From these data, a set of four selected reaction monitoring (SRM) transitions were chosen, with each identifying one compound uniquely by its transition from molecular ion to product ion in tandem mass spectrometry.

The utility of this method was tested in a LC-MS/MS system, with chromatographic conditions and mass spectrometric conditions similar to those previously described for detection of purines and urea $[10,11]$. In LC-MS/MS, injection of a drug mix resulted in a single peak for each SRM transition, although chromatographic separation among the different peaks was modest (Figure 1A). To assess the specificity of each SRM transition, 5 $\mu \mathrm{L}$ of $100 \mathrm{nM}$ of each compound was injected individually, and the signal generated from all four SRM transitions was monitored. As expected, each compound generated significant signal only within the SRM transition developed for that compound, with little or no signal from the SRM transitions for the other compounds. For example, injection of GS-9411/ P-680 resulted in signal within the SRM transition developed for that compound $(\mathrm{m} / \mathrm{z}$ $464 \rightarrow 294)$ but not SRM transitions for drug metabolites $(465 \rightarrow 171$ and $378 \rightarrow 208)$ or internal standard (449 $\rightarrow 234)$ (Figure 1B). Analysis of standard solutions revealed that detection of drug and metabolites was generally linear to concentration over several orders of magnitude (Figure 1C). Extrapolating from standard curves, limits of detection were estimated at $\sim 0.5 \mathrm{nM}$ for GS-9411/P-680 and Metabolite \#1 and $\sim 0.05 \mathrm{nM}$ for Metabolite \#2.

\subsection{EBC analysis of drug, drug metabolites, and other airway constituents}

To examine the potential of this methodology to assess airway drug concentrations in vivo, $\mathrm{EBC}$ and serum samples were collected at various time intervals before and after inhalation of GS-9411/P-680 (4 ml of a $3 \mathrm{mM}$ solution) in a study of four sheep. EBC was collected predose, immediately after dose, and 1, 2, 4, and 6 hours post dose. Serum was also collected predose and 1 and 6 hours post dose. All samples were analyzed via the LC-MS/ MS method described above which was modified to include detection of the natively occurring metabolites urea, tyrosine, phenylalanine, and adenosine [11, 17].

Because the fraction of airway secretions in EBC can vary significantly, even within the same subject, [9] a measured dilution factor is necessary to extrapolate airway concentrations from values measured in EBC. We chose to use dilution factors based on EBC:serum urea ratios, an established methodology [13] that we have used successfully in previous studies [10]. Airway concentrations of the drug, its metabolites, and other 
compounds were determined by applying these dilution factors to the concentrations measured in $\mathrm{EBC}$.

Of the drug compounds monitored, Metabolite \#2 was the most readily detected in EBC and could be assessed in all 20 EBC samples obtained post inhalation (5 time points each from 4 sheep). GS-9411/P-680 and Metabolite \#1 were below detection limits in 7 of 20 and 9 of 20 post inhalation samples respectively. Urea, tyrosine, phenylalanine, and adenosine were detected in all samples. EBC samples from one animal had low $(<1.5 \mathrm{nM})$ concentrations of all drug compounds in all post-dose samples, although EBC urea and other metabolite concentrations were similar to those measured in the other samples. Of note, measured EBC urea concentrations were highly variable (range 4.3 to $600 \mu \mathrm{M}$ ), suggesting significant sample-to-sample variation in the dilution of airway secretions within EBC, similar to that observed in previous human studies $[9,10,13,18]$.

Airway concentrations of GS-9411/P-680, Metabolite \#1, and Metabolite \#2 calculated using EBC:serum urea based dilution factors were highest 1 hour after inhalation and were still detectable 6 hours post inhalation (Figure 2A, C, E). In contrast, in the serum no significant signal for GS-9411/P-680 or Metabolite \#2 was observed in any sample. Peak concentrations of Metabolite \#1 were detected 1 hour post inhalation, and were $\sim 60$-fold lower than those in the airway (Figure 2, B, D, F, note different scales). Airway concentrations of other compounds known to be present in airway secretions did not vary significantly over the course of this study (Figure 3). To assess relationships between airway and serum drug concentrations, we calculated total drug concentration (GS-9411/P-680 plus both metabolites) at the peak one hour time point in both airway and serum samples for each animal. We observed that animals with lower serum drug concentrations also had lower airway drug concentrations, with a trend towards linear relationship between serum and airway drug concentrations ( $r=0.85, p=0.15$, Figure 4$)$.

\subsection{EBC analysis of electrolytes}

Because GS-9411/P-680 is an epithelial sodium channel blocker that may alter the electrolytes in airway surface liquid [16], we analyzed electrolyte composition of EBC and serum. EBC and serum concentrations of sodium $\left(\mathrm{Na}^{+}\right)$and potassium $\left(\mathrm{K}^{+}\right)$were successfully measured in all samples, and airway electrolyte concentrations calculated using EBC:serum urea based dilution factors. Consistent with previous observations [19, 20], airway concentrations of $\mathrm{Na}^{+}$were lower and $\mathrm{K}^{+}$higher than those measured in serum (Figure 5A-D).

Within the airway, concentrations of $\mathrm{Na}^{+}$and $\mathrm{K}^{+}$, were not significantly different at any time point. There was an interesting decline in airway sodium immediately post dose, but this change was not statistically significant $(\mathrm{p}=0.37)$ and was not maintained over time. In serum we observed a decreased serum $\mathrm{K}^{+}$over time (Figure 5D) with a trend towards increasing serum $\mathrm{Na}^{+}$(Figure 5B).

\section{Discussion}

This study demonstrates the feasibility of using EBC analysis to assess the concentrations and pharmacodynamic effects of drugs targeted towards airway surfaces. Using mass spectrometric analysis of EBC, we were able to determine the concentrations of the investigational epithelial sodium channel blocker GS-9411/P-680 and its major metabolites on airway surfaces and how these concentrations changed over time. Airway surface concentrations of parent drug and both measured metabolites peaked approximately one hour after nebulization, but were still detectable at six hours. This pattern appeared specific for the delivered drug, since concentrations of native metabolites including adenosine, 
phenylalanine, and tyrosine remained relatively constant over this time frame. Interestingly, we did observe a relationship between serum and airway peak drug concentrations.

Although not statistically significant, this finding suggests a correlation between airway concentrations and the fraction of drug delivered.

Both the concentrations and the pattern of drug relative to metabolites differed significantly between airway and serum samples. Airway drug concentrations were much higher than those detected in serum, which is consistent with the nebulized route of drug delivery. More interestingly, while the parent drug and both major drug metabolites were detected in airway samples, only one of the one drug metabolites was detected in serum. This result demonstrates that the pattern of drug metabolites in serum may not accurately reflect drug metabolism on airways surfaces. Pharmacokinetic analysis of airway drugs requires direct assessment of drug concentrations on airway surfaces, and EBC analysis provides the ability to measure such concentrations longitudinally without disturbing the airway.

Although we predicted that administration of the epithelial sodium channel blocker GS-9411/P-680 would alter airway electrolyte concentrations, we did not observe any significant effects. Interestingly, we did observe changes in serum electrolytes, with reduced potassium developing over time. This contrasts with the potassium sparing effects of systemic epithelial sodium channel blockers such as amiloride, which tend to cause hyperkalemia due primarily to a block in renal potassium secretion [21]. This discrepancy may reflect the route of delivery, with high concentrations present in the airway yet minimal systemic concentrations that could affect renal handling of electrolytes. This finding raises the possibility that the impact of drugs delivered directly to airway surfaces may differ significantly from those same agents delivered systemically. However, we interpret the findings cautiously since the observed changes in serum $\mathrm{K}$ could also reflect withholding of a high $\mathrm{K}$ diet during the course of the study.

This study was designed as a pilot study to demonstrate whether quantification of drug in EBC was feasible. The interpretation of results was somewhat limited by the small sample size the high degree of variability in airway concentrations. It is likely that some of this variability reflects the technical challenges of accurately measuring small molecules and electrolytes at the low concentrations found in EBC. However, we have also observed significant variability of airway biomarkers in other studies [10, 18], suggesting more inherent physiological variability on airway surfaces.

Although this was an animal study, the principal of assessing airway drug delivery and metabolism through analysis of EBC should be readily translated to humans. EBC collection is simple and non-invasive in human subjects, and we have routinely collected EBC volumes similar to those analyzed in this study from adults and children [10, 18][22]. Furthermore, the mass spectrometric procedure used in this study was based on the method originally developed to analyze EBC from human subjects [11] and used successfully in many studies $[10,18,22]$. These findings all suggest that application of this methodology to human trials should be straightforward.

\section{Conclusions}

In summary, this pilot study demonstrates that EBC analysis is a potentially valuable tool in assessing the pharmacokinetics of drugs on airway surfaces. The versatility of mass spectrometry suggests that this approach may be applicable to a large number of airway surface drugs, and the ease with which EBC can be collected would allow this methodology to be easily incorporated into drug development studies. 


\section{Acknowledgments}

The authors would like to thank Juan Sabater and William Abraham at Mount Sinai Medical Center at University of Miami for assistance with the animal studies.

This study was supported by a grant from Gilead Sciences, Inc. CRE was supported by NIH/NHLBI 1K23HL089708, and NIH/NIEHS P30ES10126. RCB was supported by NIH/NHLBI HL34322, HL107168, 1-P01HL08808-01A1, 2-P30-DK065988, 1-P01-HL110873-01, and 5 P50HL 107168-01.

\section{References}

1. Davis PB. Cystic Fibrosis Since 1938. Am J Respir Crit Care Med. 2006; 173:475-82. [PubMed: 16126935]

2. Barnes PJ, Chowdhury B, Kharitonov SA, Magnussen H, Page CP, Postma D, et al. Pulmonary Biomarkers in COPD. Am J Respir Crit Care Med. 2006

3. Gibson RL, Retsch-Bogart GZ, Oermann C, Milla C, Pilewski J, Daines C, et al. Microbiology, safety, and pharmacokinetics of aztreonam lysinate for inhalation in patients with cystic fibrosis. Pediatr Pulmonol. 2006; 41:656-65. [PubMed: 16703579]

4. Rosenfeld M, Gibson R, McNamara S, Emerson J, McCoyd KS, Shell R, et al. Serum and lower respiratory tract drug concentrations after tobramycin inhalation in young children with cystic fibrosis. J Pediatr. 2001; 139:572-7. [PubMed: 11598606]

5. Schwarz K, Biller H, Windt H, Koch W, Hohlfeld JM. Characterization of exhaled particles from the healthy human lung--a systematic analysis in relation to pulmonary function variables. J Aerosol Med Pulm Drug Deliv. 2010; 23:371-9. [PubMed: 20500095]

6. Johnson GR, Morawska L. The mechanism of breath aerosol formation. J Aerosol Med Pulm Drug Deliv. 2009; 22:229-37. [PubMed: 19415984]

7. Tiddens H, AWM, Donaldson SH, Rosenfeld M, Paré PD. Cystic fibrosis lung disease starts in the small airways: Can we treat it more effectively? Pediatric Pulmonology. 2010; 45:107-17. [PubMed: 20082341]

8. Hogg JC, Chu F, Utokaparch S, Woods R, Elliott WM, Buzatu L, et al. The nature of small-airway obstruction in chronic obstructive pulmonary disease. N Engl J Med. 2004; 350:2645-53. [PubMed: 15215480]

9. Effros RM, Dunning MB 3rd, Biller J, Shaker R. The promise and perils of exhaled breath condensates. Am J Physiol Lung Cell Mol Physiol. 2004; 287:L1073-80. [PubMed: 15531756]

10. Esther CR Jr, Boysen G, Olsen BM, Collins LB, Ghio AJ, Swenberg JW, et al. Mass spectrometric analysis of biomarkers and dilution markers in exhaled breath condensate reveals elevated purines in asthma and cystic fibrosis. Am J Physiol Lung Cell Mol Physiol. 2009; 296:L987-93. [PubMed: 19304910]

11. Esther CR Jr, Jasin HM, Collins LB, Swenberg JA, Boysen G. A mass spectrometric method to simultaneously measure a biomarker and dilution marker in exhaled breath condensate. Rapid Commun Mass Spectrom. 2008; 22:701-5. [PubMed: 18257110]

12. Baraldi E, Giordano G, Pasquale MF, Carraro S, Mardegan A, Bonetto G, et al. 3-Nitrotyrosine, a marker of nitrosative stress, is increased in breath condensate of allergic asthmatic children. Allergy. 2006; 61:90-6. [PubMed: 16364162]

13. Effros RM, Biller J, Foss B, Hoagland K, Dunning MB, Castillo D, et al. A simple method for estimating respiratory solute dilution in exhaled breath condensates. Am J Respir Crit Care Med. 2003; 168:1500-5. [PubMed: 14512268]

14. Sabater JR, Lee TA, Abraham WM. Comparative effects of salmeterol, albuterol, and ipratropium on normal and impaired mucociliary function in sheep. Chest. 2005; 128:3743-9. [PubMed: 16304342]

15. Hirsh AJ, Sabater JR, Zamurs A, Smith RT, Paradiso AM, Hopkins S, et al. Evaluation of second generation amiloride analogs as therapy for cystic fibrosis lung disease. J Pharmacol Exp Ther. 2004; 311:929-38. [PubMed: 15273255]

16. Hirsh AJ, Zhang J, Zamurs A, Fleegle J, Thelin WR, Caldwell RA, et al. Pharmacological properties of $\mathrm{N}$-(3,5-diamino-6-chloropyrazine-2-carbonyl)- $\mathrm{N}^{\prime}-4-[4-(2,3-$ dihydroxypropoxy) 
phenyl]butyl-guanidine methanesulfonate (552-02), a novel epithelial sodium channel blocker with potential clinical efficacy for cystic fibrosis lung disease. J Pharmacol Exp Ther. 2008; 325:77-88. [PubMed: 18218832]

17. Esther CR Jr, Peden DB, Alexis NE, Hernandez ML. Airway purinergic responses in healthy, atopic nonasthmatic, and atopic asthmatic subjects exposed to ozone. Inhal Toxicol. 2011; 23:32430. [PubMed: 21605007]

18. Esther CR Jr, Lazaar AL, Bordonali E, Qaqish B, Boucher RC. Elevated airway purines in chronic obstructive pulmonary disease. Chest. 2011; 140:954-60. [PubMed: 21454402]

19. Song Y, Thiagarajah J, Verkman AS. Sodium and chloride concentrations, $\mathrm{pH}$, and depth of airway surface liquid in distal airways. J Gen Physiol. 2003; 122:511-9. [PubMed: 14557401]

20. Jayaraman S, Song Y, Vetrivel L, Shankar L, Verkman AS. Noninvasive in vivo fluorescence measurement of airway-surface liquid depth, salt concentration, and pH. J Clin Invest. 2001; 107:317-24. [PubMed: 11160155]

21. Perazella MA. Drug-induced hyperkalemia: old culprits and new offenders. The American Journal of Medicine. 2000; 109:307-14. [PubMed: 10996582]

22. Patel K, Davis SD, Johnson R, Esther CR Jr. Exhaled breath condensate purines correlate with lung function in infants and preschoolers. Pediatr Pulmonol. 2012 
A
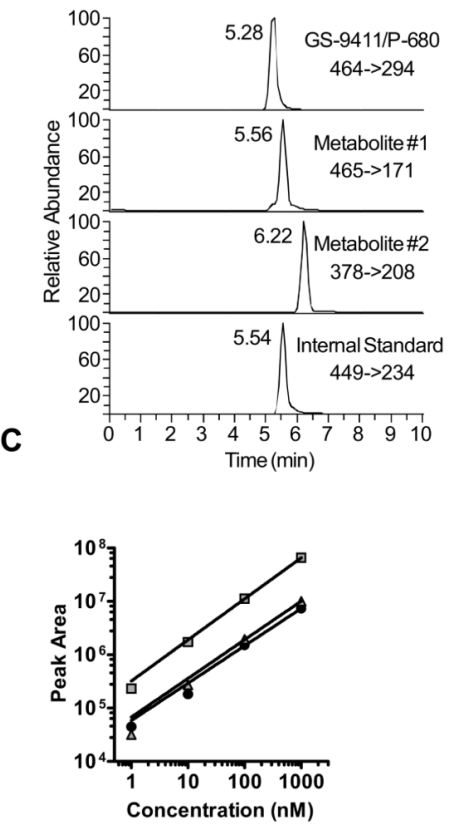

B

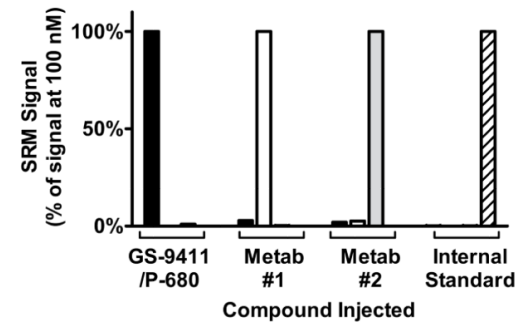

Figure 1.

Mass spectrometric detection of drug compounds. A. Mass spectrometric chromatogram demonstrating detection of GS-9411/P-680, its primary metabolites Metabolite \#1 and Metabolite \#2, and an inactive compound as an internal standard. SRM transitions for detection were $m / z$ 464 $\rightarrow 294,465 \rightarrow 171,378 \rightarrow 208$, and $449 \rightarrow 234$ respectively. Each compound was injected at $100 \mathrm{nM}$ concentration. B. Detection specificity was assessed by measuring signal generated by the SRM transitions developed for GS-9411/P-680 (black bars), Metabolite (Metab) \#1 (white bars), Metabolite \#2 (grey bars) and Internal Standard (hatched bars) after injection of $100 \mathrm{nM}$ of each compound individually. Each compound generated significant signal only within the SRM transition developed for that compound, with no meaningful signal from the SRM transitions developed for the other compounds. C. Peak areas of GS-9411/P680 (circles), Metabolite \#1 (triangles), and Metabolite \#2 (squares) were linear to concentration over several orders of magnitude. 
A

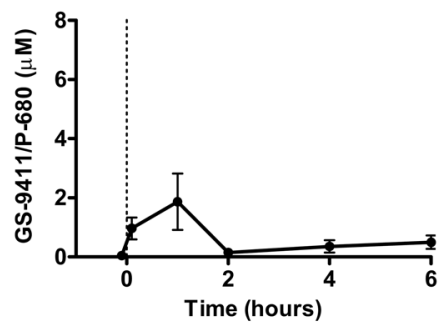

C

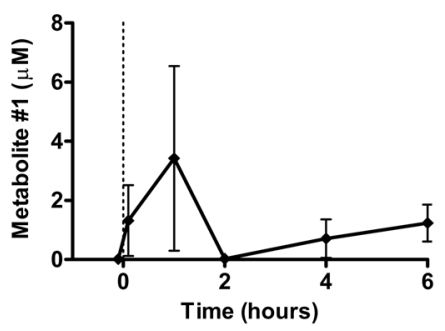

E

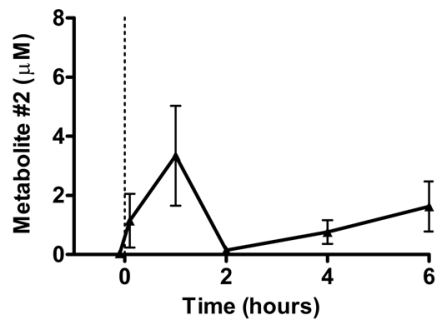

B

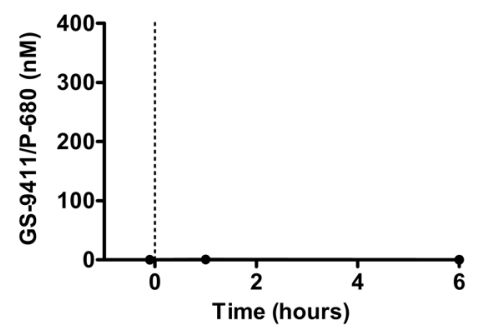

D

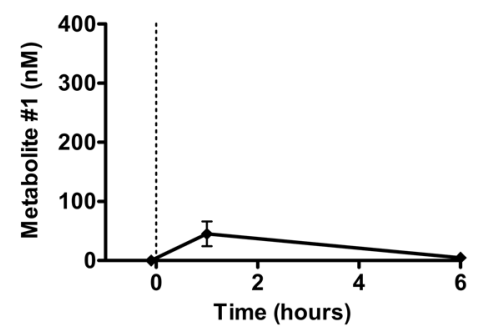

$\mathbf{F}$

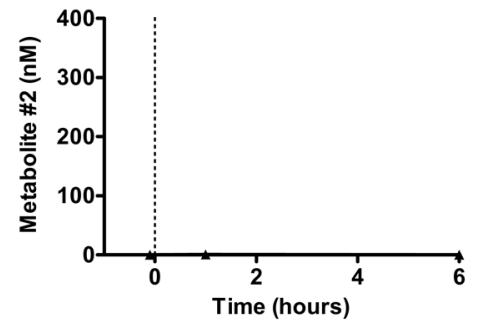

Figure 2.

Airway and serum drug concentrations. Concentrations of GS-9411/P-680 (A,B), Metabolite \#1 (C,D), and Metabolite \#2 (E,F) in the airway (A,C,E) and serum (B,D,F) were assessed after nebulization of $4 \mathrm{ml}$ of $3 \mathrm{mM}$ GS-9411/P-680 at time 0 . In the airway, drug concentrations peaked 1 hour after nebulization but were still detectable at 6 hours. Both parent compound (GS-9411/P-680) and the two principal metabolites (Metabolite \#1, Metabolite \#2) were detected. In the serum, only the Metabolite \#1 was detected at an average concentration $\sim 60$-fold lower than average airway concentration (note different scales). 
A

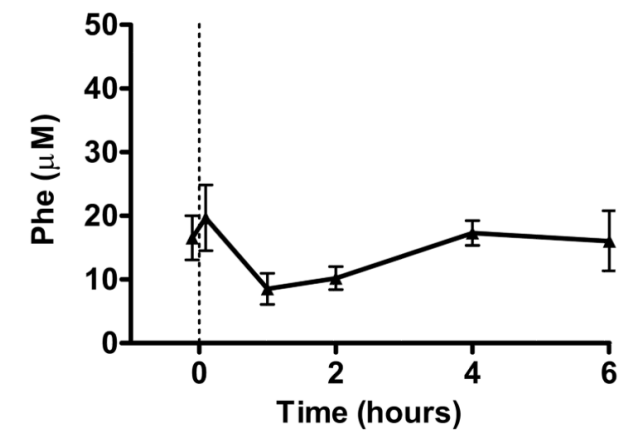

B

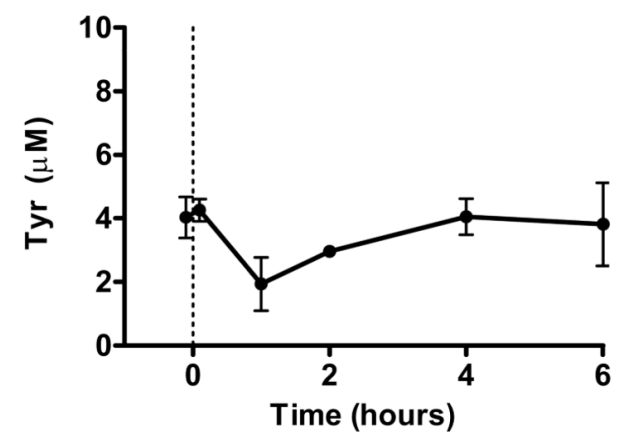

C

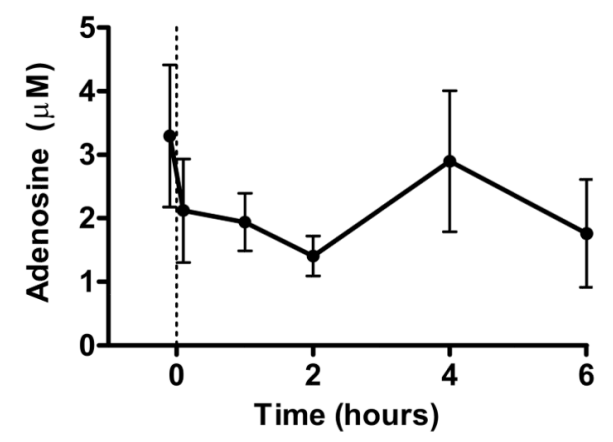

Figure 3.

Airway concentrations of endogenous compounds. Airway concentrations of phenylalanine (Phe, A), tyrosine (Tyr, B), and adenosine (C) were assessed at various time points before and after inhalation of GS-9411/P-680. No significant changes in concentrations of any compound were observed over the time frame of this study. 


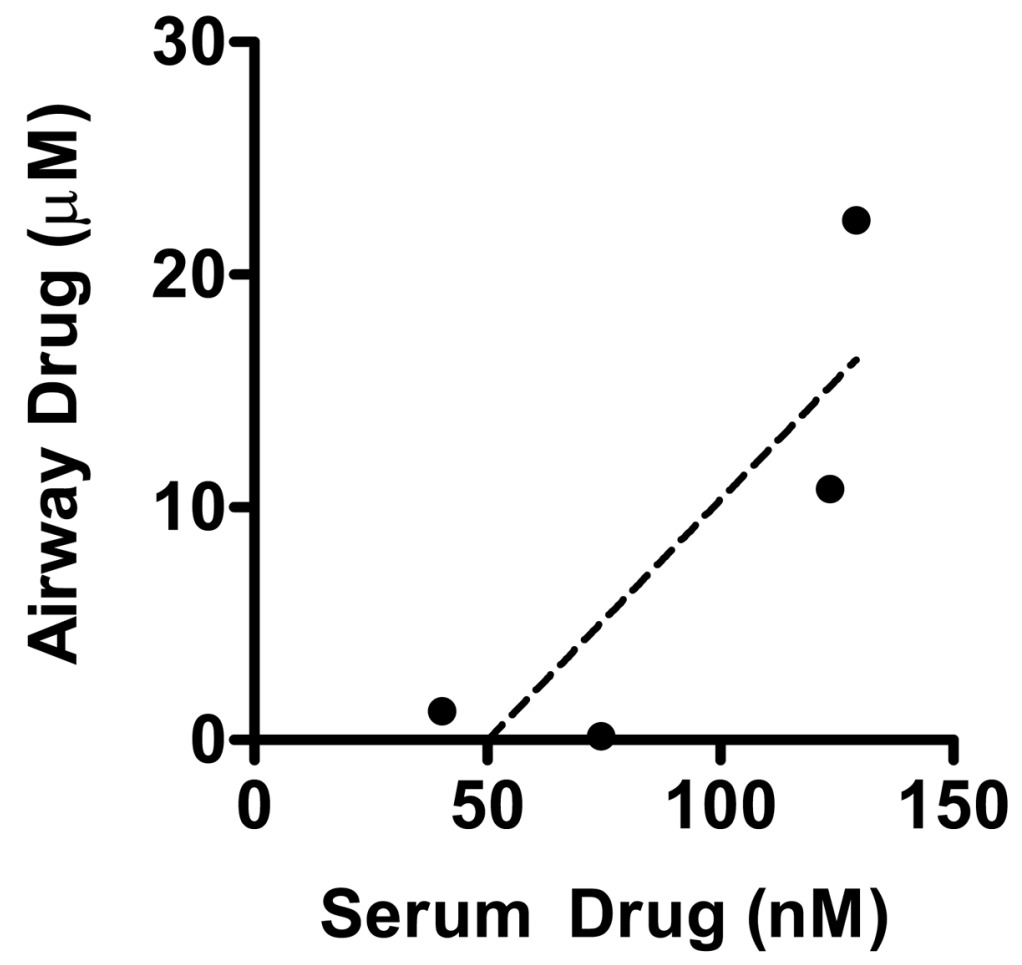

Figure 4.

Relationship between serum and airway drug concentrations. Total drug concentrations (GS-9411/P-680 + Metabolite \#1 + Metabolite \#2) were assessed at the one hour time point in both serum and airway secretions for each animal. A trend towards correlation was observed between serum and airway drug concentrations $(\mathrm{r}=0.85, \mathrm{p}=0.15)$. 
A

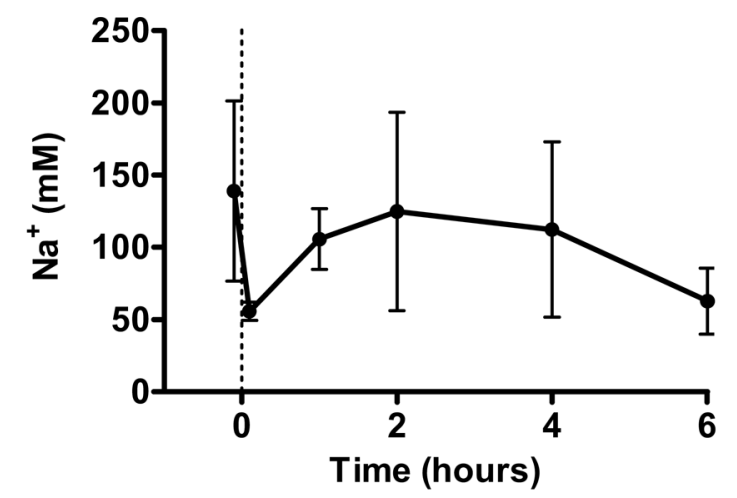

C

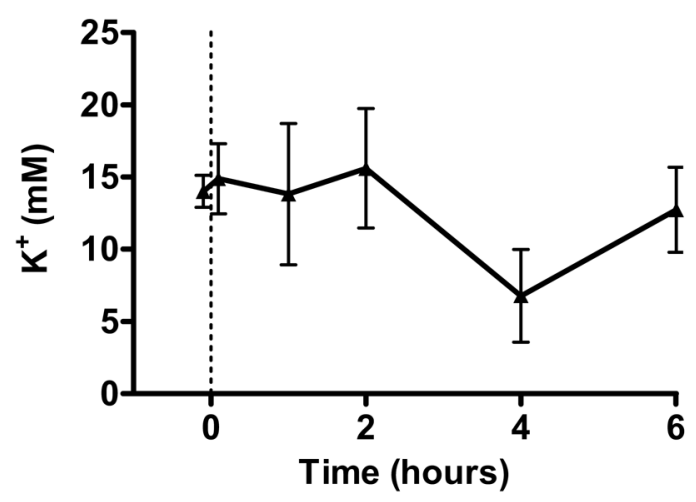

B

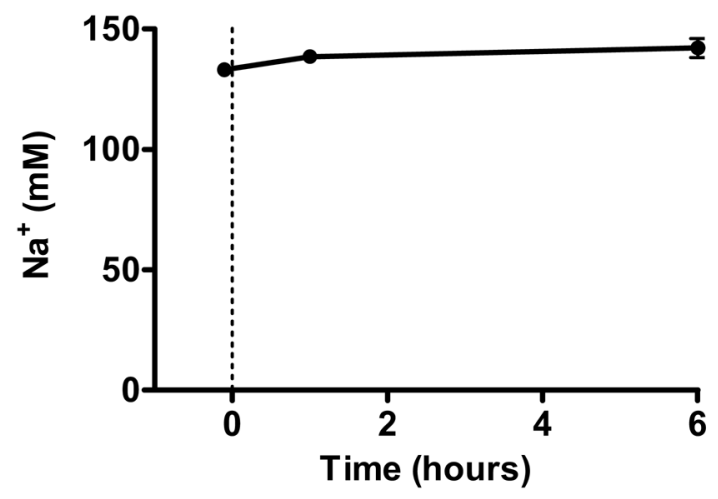

D

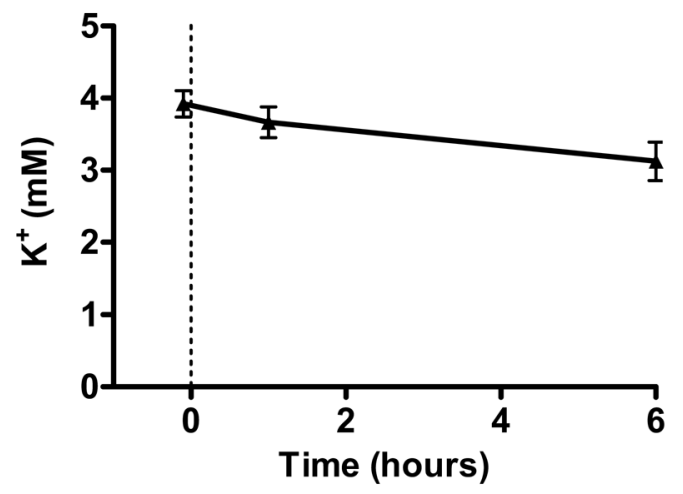

Figure 5.

Airway and serum electrolyte concentrations. Concentrations of sodium $(\mathrm{Na})(\mathrm{A}, \mathrm{B})$, potassium $(K)(C, D)$ were assessed in the airway $(A, C)$ and serum $(B, D)$ after nebulization of GS-9411/P-680 at time 0. Relative to serum, airway concentrations of Na were modestly lower and $\mathrm{K}$ significantly higher (note different scales). In the airway, there were no significant changes in any electrolyte measure, although there was a modest trend towards decreasing airway potassium over time $(\mathrm{r}=0.31, \mathrm{p}=0.14)$. In serum, $\mathrm{K}$ decreased $(\mathrm{r}=0.64$, $\mathrm{p}<0.05)$ over time, with a trend towards increasing serum $\mathrm{Na}(\mathrm{r}=0.50, \mathrm{p}=0.10)$. 
Table 1

Mass spectrometric conditions for detection of drug compounds

\begin{tabular}{|l|c|c|c|}
\hline Compound & Molecular ion $(\boldsymbol{m} / \mathbf{z})$ & Product ion $(\boldsymbol{m} / \mathbf{z})$ & Collision energy $(\mathbf{v})$ \\
\hline GS-9411/P-680 & 464.4 & 294.4 & 26 \\
\hline Metabolite \#1 & 465.3 & 171.1 & 31 \\
\hline Metabolite \#2 & 378.0 & 208.2 & 26 \\
\hline Internal Standard & 449.1 & 234.1 & 36 \\
\hline
\end{tabular}

